\title{
Collocation method with quintic b-spline method for solving hirota-satsuma coupled KDV equation
}

\author{
K. R. Raslan ${ }^{1 *}$, Talaat S. El-Danaf ${ }^{2}$, Khalid K. Ali ${ }^{1}$ \\ ${ }^{1}$ Mathematics Department, Faculty of Science, Al-Azhar University, Nasr-City, Cairo, Egypt \\ ${ }^{2}$ Mathematics Department, Faculty of Science, Menoufia University, Shebein El-Koom, Egypt \\ *Corresponding author E-mail: khalidkaram2012@yahoo.com
}

\begin{abstract}
In the present paper, a numerical method is proposed for the numerical solution of a coupled system of KdV (CKdV) equation with appropriate initial and boundary conditions by using collocation method with quintic B-spline on the uniform mesh points. The method is shown to be unconditionally stable using von-Neumann technique. To test accuracy the error norms, are computed. Three invariants of motion are predestined to determine the preservation properties of the problem, and the numerical scheme leads to careful and active results. Furthermore, interaction of two and three solitary waves is shown. These results show that the technique introduced here is easy to apply. We make linearization for the nonlinear term.
\end{abstract}

Keywords: Collocation Method; Quintic B-Splines Method; Coupled KDV Equation

\section{Introduction}

The coupled Korteweg-de Vries equation (CKdV) has introduced in 1981, by Hirota and Satsuma [1] as follows:

$$
\begin{aligned}
& u_{t}-a\left(u_{x x x}+6 u u_{x}\right)-2 b v_{x}=0, \\
& v_{t}+v_{x x x}+3 u v_{x}=0 .
\end{aligned}
$$

Where and $\mathrm{b}$ are arbitrary constants and subscripts $\mathrm{x}$ and $\mathrm{t}$ denote differentiation $x$ distance and $t$ time, is considered.

Boundary conditions

$$
\begin{array}{ll}
\mathrm{u}(\mathrm{a}, \mathrm{t})=\mathrm{f}_{1}(\mathrm{a}, \mathrm{t}), & \mathrm{u}(\mathrm{b}, \mathrm{t})=\mathrm{f}_{2}(\mathrm{~b}, \mathrm{t}), \\
\mathrm{v}(\mathrm{a}, \mathrm{t})=\mathrm{g}_{1}(\mathrm{a}, \mathrm{t}), & \mathrm{v}(\mathrm{b}, \mathrm{t})=\mathrm{g}_{2}(\mathrm{~b}, \mathrm{t}), \quad 0 \leq \mathrm{t} \leq \mathrm{T} . \\
\mathrm{u}_{\mathrm{X}}(\mathrm{a}, \mathrm{t})=\mathrm{f}_{3}(\mathrm{a}, \mathrm{t}), & \mathrm{u}_{\mathrm{X}}(\mathrm{b}, \mathrm{t})=\mathrm{f}_{4}(\mathrm{~b}, \mathrm{t}), \\
\mathrm{v}_{\mathrm{X}}(\mathrm{a}, \mathrm{t})=\mathrm{g}_{3}(\mathrm{a}, \mathrm{t}), & \mathrm{v}_{\mathrm{X}}(\mathrm{b}, \mathrm{t})=\mathrm{g}_{4}(\mathrm{~b}, \mathrm{t}), \quad 0 \leq \mathrm{t} \leq \mathrm{T} .
\end{array}
$$

And initial conditions.

$\mathrm{u}(\mathrm{x}, 0)=\mathrm{f}(\mathrm{x})$,

$\mathrm{v}(\mathrm{x}, 0)=\mathrm{g}(\mathrm{x}), \quad \mathrm{a} \leq \mathrm{x} \leq \mathrm{b}$

The CKdV has been also discussed numerically by many researchers; Halim et al. [2], [3] have studied a numerical scheme for CKdV systems. Ismail [4] discussed this system by using collocation method and quintic splines but he hasn't make linearization of the nonlinear term, in this paper we solving the CKdV equation by the same method but we take linearization of the nonlinear term we will see this linearization in section 3. Kaya and Inan [5] studied this system by using Adomian decomposition method. M. S. Ismail and H. A. Ashi, used a numerical solution for Hirota-Satsuma CKdV Equation [6]. Assas [7] used variational iteration method for solving this system. Abbasbandy [8] discussed the CKdV equation by using homotopy analysis method. Wazwaz [9] produced a finite difference scheme for solving the CKdV system. Kutluay and Ucar [10] solved the CKdV equation by using a quadratic B-spline Galerkin approach. The numerical solutions of coupled nonlinear systems are very important in applied science, for example, the coupled nonlinear Schrodinger equation which admits soliton solution and it has many applications in communication and optical fibers; this system has been discussed numerically by Ismail using finite difference and finite element methods [11-13]. A finite element algorithm based on the collocation method with trial functions taken as quintic B-spline functions over the elements will be constructed. The quintic Bspline basis together with finite element methods are shown to provide very accurate solutions in solving some partial differential equations and have been used before by several authors. In this article we are going to derive a numerical solution of the CKdV equations and to study the behavior of this solution for different values of $a$ and $b$. The brief outline of this paper is as follows. In Section 2, quintic B-spline collocation scheme is explained. In Sections 3 and 4, the method is described and applied to the CKdV equation. In Section 5, stability of the method is discussed. In Section 6, numerical examples are included to establish the applicability and accuracy of the proposed method computationally. Conclusion is given in Section 7 that briefly summarizes the numerical outcomes.

\section{Quintic b-spline functions}

To construct numerical solution, consider nodal points $\left(\mathrm{x}_{\mathrm{j}}, \mathrm{t}_{\mathrm{n}}\right)$ defined in the region $[\mathrm{a}, \mathrm{b}] \times[0, \mathrm{~T}]$ where 


$$
\begin{aligned}
& \mathrm{a}=\mathrm{x}_{0}<\mathrm{x}_{1}<\ldots<\mathrm{x}_{\mathrm{N}}=\mathrm{b}, \\
& \mathrm{h}=\mathrm{x}_{\mathrm{j}+1}-\mathrm{x}_{\mathrm{j}}=\frac{\mathrm{b}-\mathrm{a}}{\mathrm{N}}, \quad \mathrm{j}=0,1, \ldots, \mathrm{N} . \\
& 0=\mathrm{t}_{0}<\mathrm{t}_{1}<\ldots<\mathrm{t}_{\mathrm{n}}<\ldots<\mathrm{T}, \\
& \mathrm{t}_{\mathrm{n}}=\mathrm{n} \Delta \mathrm{t}, \quad \mathrm{n}=0,1, \ldots . .
\end{aligned}
$$

The quintic B-spline basis functions at knots are given by:

$$
B_{j}(x)=\frac{1}{h^{5}}\left\{\begin{array}{lc}
\left(x-x_{j}-3\right)^{5}, & x_{j-3} \leq x \leq x_{j-2} \\
\left(x-x_{j}-3\right)^{5}-6\left(x-x_{j}-2\right)^{5}, & x_{j-2} \leq x \leq x_{j}-1 \\
\left(x-x_{j}-3\right)^{5}- & \\
6\left(x-x_{j}-2\right)^{5}+ & x_{j-1} \leq x \leq x_{j} \\
15\left(x-x_{j-1}\right)^{5}, & \\
\left(-x+x_{j+3}\right)^{5}+ & x_{j} \leq x \leq x_{j+1} \\
6\left(x-x_{j+2}\right)^{5}- & x_{j+1} \leq x \leq x_{j+2} \\
15\left(x-x_{j+1}\right)^{5}, & x_{j+2} \leq x \leq x_{j+3} \\
\left(-x+x_{j+3}\right)^{5}+ & \text { otherwise } \\
6\left(x-x_{j+2}\right)^{5}, &
\end{array}\right.
$$

Using quintic B-spline basis function (5) the values of $B_{j}(x)$ and its derivatives at the knots points can be calculated, which are tabulated in Table 1.

\section{Solution of CKdV equation}

To apply the proposed method, we rewrite (1) and (2) as

$$
\begin{aligned}
& \frac{\partial \mathrm{u}(\mathrm{x}, \mathrm{t})}{\partial \mathrm{t}}-\mathrm{a}\left(\frac{\partial \mathrm{u}(\mathrm{x}, \mathrm{t})}{\partial \mathrm{x}^{3}}+6 \mathrm{u}(\mathrm{x}, \mathrm{t}) \frac{\partial \mathrm{u}(\mathrm{x}, \mathrm{t})}{\partial \mathrm{x}}\right)- \\
& 2 \mathrm{~b}\left[\mathrm{v}(\mathrm{x}, \mathrm{t}) \frac{\partial \mathrm{v}(\mathrm{x}, \mathrm{t})}{\partial \mathrm{x}}\right]=0, \\
& \frac{\partial \mathrm{v}(\mathrm{x}, \mathrm{t})}{\partial \mathrm{t}}+\frac{\partial \mathrm{v}(\mathrm{x}, \mathrm{t})}{\partial \mathrm{x}^{3}}+3 \mathrm{u}(\mathrm{x}, \mathrm{t}) \frac{\partial \mathrm{v}(\mathrm{x}, \mathrm{t})}{\partial \mathrm{x}}=0,
\end{aligned}
$$

We take the approximations $u(x, t)=U_{j}^{n}$ and $v(x, t)=V_{j}^{n}$, then from famous Cranck-Nicolson scheme and forward finite difference approximation for the derivative $t,[14]$. We get

$$
\begin{aligned}
& \frac{U_{j}^{n+1}-U_{j}^{n}}{k}=a\left[\begin{array}{l}
\frac{U_{x x x_{j}^{n+1}}+U_{x x x} n_{j}}{2}+ \\
\frac{\left(U U_{x}\right)_{j}^{n+1}+\left(U U_{x}\right)_{j}^{n}}{2}
\end{array}\right]+ \\
& 2 b\left[\frac{\left(V V_{x}\right)_{j}^{n+1}+\left(V V_{x}\right)_{j}^{n}}{2}\right], \\
& \frac{V_{j}^{n+1}-V_{j}^{n}}{k}=-\left[\frac{V_{x x x}{ }_{j}^{n+1}+V_{x x x} n}{2}\right]- \\
& 3\left[\frac{\left(U V_{x}\right)_{j}^{n+1}+\left(U V_{x}\right)_{j}^{n}}{2}\right],
\end{aligned}
$$

Where $\mathrm{k}=\Delta \mathrm{t}$ is the time step?

Table 1: The Values of Quintic B-Spline and Its First and Second Derivatives at the Knots Points

\begin{tabular}{cccccccc}
\hline $\mathrm{x}$ & $\mathrm{x}_{\mathrm{j}-3}$ & $\mathrm{x}_{\mathrm{j}-2}$ & $\mathrm{x}_{\mathrm{j}-1}$ & $\mathrm{x}_{\mathrm{j}}$ & $\mathrm{x}_{\mathrm{j}+1}$ & $\mathrm{x}_{\mathrm{j}+2}$ & $\mathrm{x}_{\mathrm{j}+3}$ \\
\hline $\mathrm{B}_{\mathrm{j}}$ & 0 & 1 & 26 & 66 & 26 & 1 & 0 \\
$\mathrm{~B}_{\mathrm{j}}^{\prime}$ & 0 & $\frac{-5}{\mathrm{~h}}$ & $\frac{-50}{\mathrm{~h}}$ & 0 & $\frac{50}{\mathrm{~h}}$ & $\frac{5}{\mathrm{~h}}$ & 0 \\
$\mathrm{~B}_{\mathrm{j}}^{\prime \prime}$ & 0 & $\frac{20}{\mathrm{~h}^{2}}$ & $\frac{40}{\mathrm{~h}^{2}}$ & $\frac{-120}{\mathrm{~h}^{2}}$ & $\frac{40}{\mathrm{~h}^{2}}$ & $\frac{20}{\mathrm{~h}^{2}}$ & 0 \\
$\mathrm{~B}_{\mathrm{j}}^{\prime \prime \prime}$ & 0 & $\frac{-60}{\mathrm{~h}^{3}}$ & $\frac{120}{\mathrm{~h}^{3}}$ & 0 & $-\frac{120}{\mathrm{~h}^{3}}$ & $\frac{60}{\mathrm{~h}^{3}}$ & 0 \\
\hline
\end{tabular}

In the Crank-Nicolson scheme, the time stepping process is half explicit and half implicit. So the method is better than simple finite difference method.

The nonlinear terms in Eqs. (6) and (7) is linearized using the form given by Rubin and Graves [15] as: we take linearization of the nonlinear term as follows

$$
\begin{aligned}
& \left(U V_{x}\right)_{j}^{n+1}=U_{j}^{n} V_{x_{j}}^{n+1}+U_{j}^{n+1} V_{x_{j}}^{n}-U_{j}^{n} V_{x_{j}}^{n}, \\
& \left(V V_{x}\right)_{j}^{n+1}=V_{j}^{n} V_{x_{j}}^{n+1}+V_{j}^{n+1} V_{x_{j}}^{n}-V_{j}^{n} V_{x_{j}}^{n}, \\
& \left(U U_{x}\right)_{j}^{n+1}=U_{j}^{n} U_{x_{j}}^{n+1}+U_{j}^{n+1} U_{x_{j}}^{n}-U_{j}^{n} U_{x_{j}}^{n}
\end{aligned}
$$

Expressing $\mathrm{U}(\mathrm{x}, \mathrm{t})$ and $\mathrm{V}(\mathrm{x}, \mathrm{t})$ by using quintic $\mathrm{B}$-spline functions $\mathrm{B}_{\mathrm{j}}(\mathrm{x})$ and the time dependent parameters $\mathrm{c}_{\mathrm{j}}(\mathrm{t})$ and $\delta_{\mathrm{j}}(\mathrm{t})$, for $\mathrm{U}(\mathrm{x}, \mathrm{t})$ and $\mathrm{V}(\mathrm{x}, \mathrm{t})$ respectively, the approximate solution can be written as:

$U_{N}(x, t)=\sum_{j=-2}^{N+2} c_{j}(t) B_{j}(x), \quad V_{N}(x, t)=\sum_{j=-2}^{N+2} \delta_{j}(t) B_{j}(x)$,

Using approximate function (9) and quintic B-spline functions (5), the approximate values $\mathrm{U}(\mathrm{x}), \mathrm{V}(\mathrm{x})$ and their derivatives up to second order are determined in terms of the time parameters $c_{j}(t)$ and $\delta_{\mathrm{j}}(\mathrm{t})$, respectively, as

$$
\begin{aligned}
& U_{j}=U\left(x_{j}\right)=c_{j-2}+26 c_{j-1}+66 c_{j}+26 c_{j+1}+c_{j+2}, \\
& \mathrm{U}_{\mathrm{j}}^{\prime}=\mathrm{U}^{\prime}\left(\mathrm{x}_{\mathrm{j}}\right)=\frac{5}{\mathrm{~h}}\left(\mathrm{c}_{\mathrm{j}+2}+10 \mathrm{c}_{\mathrm{j}+1}-10 \mathrm{c}_{\mathrm{j}-1}-\mathrm{c}_{\mathrm{j}-2}\right) \text {, } \\
& \mathrm{U}_{j}^{\prime \prime}=\mathrm{U}^{\prime \prime}\left(\mathrm{x}_{\mathrm{j}}\right)=\frac{20}{\mathrm{~h}^{2}}\left(\mathrm{c}_{\mathrm{j}-2}+2 \mathrm{c}_{\mathrm{j}-1}-6 \mathrm{c}_{\mathrm{j}}+2 \mathrm{c}_{\mathrm{j}+1}+\mathrm{c}_{\mathrm{j}+2}\right) \text {, } \\
& \mathrm{U}_{\mathrm{j}}^{\prime \prime \prime}=\mathrm{U}^{\prime \prime \prime}\left(\mathrm{x}_{\mathrm{j}}\right)=\frac{60}{\mathrm{~h}^{3}}\left(-\mathrm{c}_{\mathrm{j}-2}+2 \mathrm{c}_{\mathrm{j}-1}-2 \mathrm{c}_{\mathrm{j}+1}+\mathrm{c}_{\mathrm{j}+2}\right), \\
& V_{\mathrm{j}}=\mathrm{V}\left(\mathrm{x}_{\mathrm{j}}\right)=\delta_{\mathrm{j}-2}+26 \delta_{\mathrm{j}-1}+66 \delta_{\mathrm{j}}+26 \delta_{\mathrm{j}+1}+\delta_{\mathrm{j}+2} \text {, } \\
& \mathrm{V}_{\mathrm{j}}^{\prime}=\mathrm{V}^{\prime}\left(\mathrm{x}_{\mathrm{j}}\right)=\frac{5}{\mathrm{~h}}\left(\delta_{\mathrm{j}+2}+10 \delta_{\mathrm{j}+1}-10 \delta_{\mathrm{j}-1}-\delta_{\mathrm{j}-2}\right), \\
& \mathrm{V}_{\mathrm{j}}^{\prime \prime}=\mathrm{V}^{\prime \prime}\left(\mathrm{x}_{\mathrm{j}}\right)=\frac{20}{\mathrm{~h}^{2}}\left(\delta_{\mathrm{j}-2}+2 \delta_{\mathrm{j}-1}-6 \delta_{\mathrm{j}}+2 \delta_{\mathrm{j}+1}+\delta_{\mathrm{j}+2}\right) \text {. } \\
& \mathrm{V}_{\mathrm{j}}^{\prime \prime \prime}=\mathrm{V}^{\prime \prime \prime}\left(\mathrm{x}_{\mathrm{j}}\right)=\frac{60}{\mathrm{~h}^{3}}\left(-\delta_{\mathrm{j}-2}+2 \delta_{\mathrm{j}-1}-2 \delta_{\mathrm{j}+1}+\delta_{\mathrm{j}+2}\right) \text {. }
\end{aligned}
$$

On substituting the approximate solution for $\mathrm{U}, \mathrm{V}$ and its derivatives from Eq. (10) at the knots in Eqs. (6) and (7) yields the following difference equation with the variables $\mathrm{c}_{\mathrm{j}}(\mathrm{t})$ and $\delta_{\mathrm{j}}(\mathrm{t})$. 


$$
\begin{aligned}
& A_{1} c_{j-2}^{n+1}+A_{2} c_{j-1}^{n+1}+A_{3} c_{j}^{n+1}+A_{4} c_{j+1}^{n+1}+ \\
& A_{5} c_{j+2}^{n+1}+A_{6} \delta_{j-2}^{n+1}+A_{7} \delta_{j-1}^{n+1}+A_{8} \delta_{j}^{n+1} \\
& +A_{9} \delta_{j+1}^{n+1}+A_{10} \delta_{j+2}^{n+1}=A_{11} c_{j-2}^{n}+ \\
& A_{12} c_{j-1}^{n}+A_{13} c_{j}^{n}+A_{14} c_{j+1}^{n}+A_{15} c_{j+2}^{n}, \\
& B_{1} \delta_{j-2}^{n+1}+B_{2} \delta_{j-1}^{n+1}+B_{3} \delta_{j}^{n+1}+B_{4} \delta_{j+1}^{n+1}+ \\
& B_{5} \delta_{j+2}^{n+1}+B_{6} c_{j-2}^{n+1}+B_{7} c_{j-1}^{n+1}+B_{8} c_{j}^{n+1} \\
& +B_{9} c_{j+1}^{n+1}+B_{10} c_{j+2}^{n+1}=A_{11} \delta_{j-2}^{n}+ \\
& A_{12} \delta_{j-1}^{n}+A_{13} \delta_{j}^{n}+A_{14} \delta_{j+1}^{n}+A_{15} \delta_{j+2}^{n},
\end{aligned}
$$

\section{Where}

$$
\begin{aligned}
& \mathrm{A}_{1}=1+\frac{30 \mathrm{a} \Delta \mathrm{t}}{\mathrm{h}^{3}}-\frac{15 \mathrm{a} \Delta \mathrm{t}}{\mathrm{h}} \mathrm{z}_{2}+\frac{15 \mathrm{a} \Delta \mathrm{t}}{\mathrm{h}} \mathrm{z}_{1}, \\
& \mathrm{~A}_{2}=26-2 \frac{30 \mathrm{a} \Delta \mathrm{t}}{\mathrm{h}^{3}}-26 \frac{15 \mathrm{a} \Delta \mathrm{t}}{\mathrm{h}} \mathrm{z}_{2}+10 \frac{15 \mathrm{a} \Delta \mathrm{t}}{\mathrm{h}} \mathrm{z}_{1}, \\
& \mathrm{~A}_{3}=66-66 \frac{15 \mathrm{a} \Delta \mathrm{t}}{\mathrm{h}} \mathrm{z}_{2}, \\
& \mathrm{~A}_{4}=26+2 \frac{30 \mathrm{a} \Delta \mathrm{t}}{\mathrm{h}^{3}}-\frac{15 \mathrm{a} \Delta \mathrm{t}}{\mathrm{h}} \mathrm{z}_{2}-10 \frac{15 \mathrm{a} \Delta \mathrm{t}}{\mathrm{h}} \mathrm{z}_{1}, \\
& \mathrm{~A}_{5}=1-\frac{30 \mathrm{a} \Delta \mathrm{t}}{\mathrm{h}^{3}}-\frac{15 \mathrm{a} \Delta \mathrm{t}}{\mathrm{h}} \mathrm{z}_{2}-\frac{15 \mathrm{a} \Delta \mathrm{t}}{\mathrm{h}} \mathrm{z}_{1}, \\
& \mathrm{~A}_{6}=-\frac{5 \mathrm{~b} \Delta \mathrm{t}}{\mathrm{h}} \mathrm{z}_{4}+\frac{5 \mathrm{~b} \Delta \mathrm{t}}{\mathrm{h}} \mathrm{z}_{3}, \\
& \mathrm{~A}_{7}=-26 \frac{5 \mathrm{~b} \Delta \mathrm{t}}{\mathrm{h}} \mathrm{z}_{4}+10 \frac{5 \mathrm{~b} \Delta \mathrm{t}}{\mathrm{h}} \mathrm{z}_{3}, \\
& \mathrm{~A}_{8}=-66 \frac{5 \mathrm{~b} \Delta \mathrm{t}}{\mathrm{h}} \mathrm{z}_{4}, \\
& \mathrm{~A}_{9}=-26 \frac{5 \mathrm{~b} \Delta \mathrm{t}}{\mathrm{h}} \mathrm{z}_{4}-10 \frac{5 \mathrm{~b} \Delta \mathrm{t}}{\mathrm{h}} \mathrm{z}_{3}, \\
& \mathrm{~A}_{12}=26+2 \frac{30 \mathrm{a} \Delta \mathrm{t}}{\mathrm{h}^{3}}, \\
& \mathrm{~A}_{10}=66, \frac{5 \mathrm{~b} \Delta \mathrm{t}}{\mathrm{h}} \mathrm{z}_{4}-\frac{5 \mathrm{~b} \Delta \mathrm{t}}{\mathrm{h}} \mathrm{z}_{3}, \\
& \mathrm{~A}_{11}=1-\frac{30 \mathrm{a} \Delta \mathrm{t}}{\mathrm{h}^{3}},
\end{aligned}
$$

$$
\begin{aligned}
& \mathrm{B}_{1}=1-\frac{30 \Delta \mathrm{t}}{\mathrm{h}^{3}}-\frac{15 \Delta \mathrm{t}}{2 \mathrm{~h}} \mathrm{z}_{1}, \\
& \mathrm{~B}_{2}=26+2 \frac{30 \Delta \mathrm{t}}{\mathrm{h}^{3}}-10 \frac{15 \Delta \mathrm{t}}{2 \mathrm{~h}} \mathrm{z}_{1}, \\
& \mathrm{~B}_{3}=66, \quad \mathrm{~B}_{4}=26-2 \frac{30 \Delta \mathrm{t}}{\mathrm{h}^{3}}+10 \frac{15 \Delta \mathrm{t}}{2 \mathrm{~h}} \mathrm{z}_{1}, \\
& \mathrm{~B}_{5}=1+\frac{30 \Delta \mathrm{t}}{\mathrm{h}^{3}}+\frac{15 \Delta \mathrm{t}}{2 \mathrm{~h}} \mathrm{z}_{1}, \\
& \mathrm{~B}_{6}=\frac{15 \Delta \mathrm{t}}{2 \mathrm{~h}} \mathrm{z}_{4}, \\
& \mathrm{~B}_{7}=26 \frac{15 \Delta \mathrm{t}}{2 \mathrm{~h}} \mathrm{z}_{4}, \\
& \mathrm{~B}_{8}=66 \frac{15 \Delta \mathrm{t}}{2 \mathrm{~h}} \mathrm{z}_{4}, \\
& \mathrm{~B}_{9}=26 \frac{15 \Delta \mathrm{t}}{2 \mathrm{~h}} \mathrm{z}_{4}, \\
& \mathrm{~B}_{10}=\frac{15 \Delta \mathrm{t}}{2 \mathrm{~h}} \mathrm{z}_{4}, \\
& \mathrm{~B}_{11}=1, \\
& \mathrm{~B}_{13}=66, \quad \mathrm{~B}_{12}=26, \\
& \mathrm{z}_{1}=\mathrm{c}_{\mathrm{j}-2}+26 \mathrm{c}_{j-1}+66 \mathrm{c}_{\mathrm{j}}+26 \mathrm{c}_{\mathrm{j}+1}+\mathrm{c}_{\mathrm{j}+2}, \\
& \mathrm{z}_{2}=\mathrm{c}_{\mathrm{j}+2}+10 \mathrm{c}_{\mathrm{j}+1}-10 \mathrm{c}_{\mathrm{j}-1}-\mathrm{c}_{\mathrm{j}-2}, \\
& \mathrm{z}_{3}=\delta_{\mathrm{j}-2}+26 \delta_{j-1}+66 \delta_{\mathrm{j}}+26 \delta_{\mathrm{j}+1}+\delta_{\mathrm{j}+2}, \\
& \mathrm{z}_{4}=\delta_{\mathrm{j}+2}+10 \delta_{j+1}-10 \delta_{j-1}-\delta_{\mathrm{j}-2},
\end{aligned}
$$

The system thus obtained on simplifying Eqs. (11) and (12) consists of $(2 \mathrm{~N}+2)$ linear equations in the $(2 \mathrm{~N}+10)$ unknowns

$$
\begin{aligned}
& \left(c_{-2}, c_{-1}, c_{0}, \ldots \ldots, c_{N}, c_{N+1}, c_{N+2}\right)^{T}, \\
& \left(\delta_{-2}, \delta_{-1}, \delta_{0}, \ldots \ldots, \delta_{N}, \delta_{N+1}, \delta_{N+2}\right)^{\mathrm{T}} .
\end{aligned}
$$

To obtain a unique solution to the resulting system four additional constraints are required. These are obtained by imposing boundary conditions. Eliminating $\mathrm{c}_{-2}, \mathrm{c}_{-1}, \mathrm{c}_{\mathrm{N}+1}, \mathrm{c}_{\mathrm{N}+2}$ and $\delta_{-2}, \delta_{-1}, \delta_{\mathrm{N}+1}, \delta_{\mathrm{N}+2}$ the system get reduced to a matrix system of dimension $(2 \mathrm{~N}+2) \times(2 \mathrm{~N}+2)$ which is the penta-diagonal system that can be solved by any algorithm.

\section{Initial values}

To find the initial parameters $\mathrm{c}_{\mathrm{j}}^{0}$ and $\delta_{\mathrm{j}}^{0}$, the initial conditions and the derivatives at the boundaries are used in the following way

$$
\begin{aligned}
& \left(U^{\prime}\right)\left(x_{0}, 0\right)=\frac{5}{h}\left(c_{2}+10 c_{1}-10 c_{-1}-c_{-2}\right)=0 \\
& \left(U^{\prime \prime}\right)\left(x_{0}, 0\right)=\frac{20}{h^{2}}\left(c_{-2}+2 c_{-1}-6 c_{0}+2 c_{j+1}+c_{j+2}\right)=0 \\
& (U)\left(x_{j}, 0\right)=c_{j-2}+26 c_{j-1}+66 c_{j}+ \\
& 26 c_{j+1}+c_{j+2}=0 \\
& \left(U^{\prime}\right)\left(x_{N}, 0\right)=\frac{5}{h}\left(c_{N+2}+10 c_{N+1}-\right. \\
& \left.10 c_{N-1}-c_{N-2}\right)=0 \\
& \left(U^{\prime \prime}\right)\left(x_{N}, 0\right)=\frac{20}{h^{2}}\left(c_{N-2}+2 c_{N-1}-6 c_{N}+\right. \\
& \left.2 c_{N+1}+c_{N+2}\right)=0 \\
& \left(V^{\prime}\right)\left(x_{0}, 0\right)=\frac{5}{h}\left(\delta_{2}+10 \delta_{1}-10 \delta_{-1}-\delta_{-2}\right)=0 \\
& \left(V^{\prime \prime}\right)\left(x_{0}, 0\right)=\frac{20}{h^{2}}\left(\delta_{-2}+2 \delta_{-1}-\right. \\
& \left.6 \delta_{0}+2 \delta_{1}+\delta_{2}\right)=0
\end{aligned}
$$


$(V)\left(x_{j}, 0\right)=\delta_{j-2}+26 \delta_{j-1}+$

$66 \delta_{j}+26 \delta_{j+1}+\delta_{j+2}=0$,

$\left(V^{\prime}\right)\left(x_{N}, 0\right)=\frac{5}{h}\left(\delta_{N+2}+10 \delta_{N+1}-\right.$

$\left.10 \delta_{N-1}-\delta_{N-2}\right)=0$,

$\left(V^{\prime \prime}\right)\left(x_{N}, 0\right)=\frac{20}{h^{2}}\left(\delta_{N-2}+2 \delta_{N-1}-6 \delta_{N}\right.$

$\left.+2 \delta_{N+1}+\delta_{N+2}\right)=0$,

Which forms a linear block pintadiagonal system for unknown initial conditions $c_{j}^{0}$ and $\delta_{j}^{0}$, of order $(2 \mathrm{~N}+2)$ after eliminating the functions values of $\mathrm{c}$ and $\delta$. This system can be solved by any algorithm. Once the initial vectors of parameters have been calculated, the numerical solution of the $\mathrm{CKdV}$ equation $\mathrm{U}$ and $\mathrm{V}$ can be determined from the time evaluation of the vectors $c_{j}^{n}$ and $\delta_{j}^{n}$, by using the recurrence relations

$$
\begin{aligned}
& \mathrm{U}\left(\mathrm{x}_{\mathrm{j}}, \mathrm{t}_{\mathrm{n}}\right)=\mathrm{c}_{\mathrm{j}-2}^{\mathrm{n}}+26 \mathrm{c}_{\mathrm{j}-1}^{\mathrm{n}}+66 \mathrm{c}_{\mathrm{j}}^{\mathrm{n}}+26 \mathrm{c}_{\mathrm{j}+1}^{\mathrm{n}}+\mathrm{c}_{\mathrm{j}+2}^{\mathrm{n}}, \\
& \mathrm{V}\left(\mathrm{x}_{\mathrm{j}}, \mathrm{t}_{\mathrm{n}}\right)=\delta_{\mathrm{j}-2}^{\mathrm{n}}+26 \delta_{\mathrm{j}-1}^{\mathrm{n}}+66 \delta_{j}^{\mathrm{n}}+26 \delta_{j+1}^{\mathrm{n}}+\delta_{\mathrm{j}+2}^{\mathrm{n}} .
\end{aligned}
$$

\section{Stability analysis of the method}

The stability analysis of nonlinear partial differential equations is not easy task to undertake. Most researchers copy with the problem by linearizing the partial differential equation. Our stability analysis will be based on the Von-Neumann concept in which the growth factor of a typical Fourier mode defined as

$c_{j}^{n}=A \zeta^{n} \exp (i j \varphi)$,

$\delta_{\mathrm{j}}^{\mathrm{n}}=\mathrm{B} \zeta^{\mathrm{n}} \exp (\mathrm{ij} \varphi)$

$\mathrm{g}=\frac{\zeta^{\mathrm{n}+1}}{\zeta^{\mathrm{n}}}$,

Where A and B are the harmonics amplitude, $\varphi=\mathrm{kh}, \mathrm{k}$ is the mode number, $i=\sqrt{-1}$ and

$\mathrm{g}$ is the amplification factor of the schemes. We will be applied the stability of the quintic schemes by assuming the nonlinear term as a constants $\lambda_{1}, \lambda_{2}$. This is equivalent to assuming that all the $c_{j}^{n}$ and $\delta_{\mathrm{j}}^{\mathrm{n}}$ as a local constants $\lambda_{1}, \lambda_{2}$ respectively. At $\mathrm{x}=\mathrm{x}_{\mathrm{j}}$ systems (11) and (12) can be written as

$$
\begin{aligned}
& a_{1} c_{j-2}^{n+1}+a_{2} c_{j-1}^{n+1}+a_{3} c_{j}^{n+1}+a_{4} c_{j+1}^{n+1}+ \\
& a_{5} c_{j+2}^{n+1}+a_{6} \delta_{j-2}^{n+1}+a_{7} \delta_{j-1}^{n+1} \\
& +a_{8} \delta_{j+1}^{n+1}+a_{9} \delta_{j+2}^{n+1}=a_{10} c_{j-2}^{n}+ \\
& a_{11} c_{j-1}^{n}+a_{1} c_{j}^{n}+a_{13} c_{j+1}^{n}+a_{14} c_{j+2}^{n} \\
& -a_{6} \delta_{j-2}^{n+1}-a_{7} \delta_{j-1}^{n+1}-a_{8} \delta_{j+1}^{n+1}-a_{9} \delta_{j+2}^{n+1},
\end{aligned}
$$

$$
\begin{aligned}
& \mathrm{a}_{1}=1+\frac{30 \mathrm{a} \Delta \mathrm{t}}{\mathrm{h}^{3}}+\frac{15 \mathrm{a} \Delta \mathrm{t}}{\mathrm{h}} \lambda_{1}, \\
& \mathrm{a}_{2}=26-\frac{60 \mathrm{a} \Delta \mathrm{t}}{\mathrm{h}^{3}}+\frac{150 \mathrm{a} \Delta \mathrm{t}}{\mathrm{h}} \lambda_{1}, \\
& \mathrm{a}_{3}=66 \text {, } \\
& \mathrm{a}_{4}=26+\frac{60 \mathrm{a} \Delta \mathrm{t}}{\mathrm{h}^{3}}-\frac{150 \mathrm{a} \Delta \mathrm{t}}{\mathrm{h}} \lambda_{1}, \\
& \mathrm{a}_{5}=1-\frac{30 \mathrm{a} \Delta \mathrm{t}}{\mathrm{h}^{3}}-\frac{15 \mathrm{a} \Delta \mathrm{t}}{\mathrm{h}} \lambda_{1}, \\
& \mathrm{a}_{6}=-\frac{5 \mathrm{~b} \Delta \mathrm{t}}{\mathrm{h}} \lambda_{2}, \\
& \mathrm{a}_{7}=-\frac{150 \mathrm{~b} \Delta \mathrm{t}}{\mathrm{h}} \lambda_{2} \text {, } \\
& \mathrm{a}_{8}=\frac{150 \mathrm{~b} \Delta \mathrm{t}}{\mathrm{h}} \lambda_{2} \text {, } \\
& \mathrm{a}_{9}=\frac{5 \mathrm{~b} \Delta \mathrm{t}}{\mathrm{h}} \lambda_{2}, \\
& \mathrm{a}_{10}=1-\frac{30 \mathrm{a} \Delta \mathrm{t}}{\mathrm{h}^{3}}-\frac{15 \mathrm{a} \Delta \mathrm{t}}{\mathrm{h}} \lambda_{1}, \\
& \mathrm{a}_{11}=26+\frac{60 \mathrm{a} \Delta \mathrm{t}}{\mathrm{h}^{3}}-\frac{150 \mathrm{a} \Delta \mathrm{t}}{\mathrm{h}} \lambda_{1}, \quad \mathrm{a}_{12}=66, \\
& \mathrm{a}_{13}=26-\frac{60 \mathrm{a} \Delta \mathrm{t}}{\mathrm{h}^{3}}+\frac{150 \mathrm{a} \Delta \mathrm{t}}{\mathrm{h}} \lambda_{1}, \\
& \mathrm{a}_{14}=1+\frac{30 \mathrm{a} \Delta \mathrm{t}}{\mathrm{h}^{3}}+\frac{15 \mathrm{a} \Delta \mathrm{t}}{\mathrm{h}} \lambda_{1}, \\
& \mathrm{~d}_{1} \delta_{\mathrm{j}-2}^{\mathrm{n}+1}+\mathrm{d}_{2} \delta_{\mathrm{j}-1}^{\mathrm{n}+1}+\mathrm{d}_{3} \delta_{j}^{\mathrm{n}+1}+\mathrm{d}_{4} \delta_{\mathrm{j}+1}^{\mathrm{n}+1}+\mathrm{d}_{5} \delta_{\mathrm{j}+2}^{\mathrm{n}+1}= \\
& \mathrm{d}_{5} \delta_{\mathrm{j}-2}^{\mathrm{n}}+\mathrm{d}_{4} \delta_{\mathrm{j}-1}^{\mathrm{n}}+\mathrm{d}_{3} \delta_{\mathrm{j}}^{\mathrm{n}}+\mathrm{d}_{2} \delta_{\mathrm{j}+1}^{\mathrm{n}}+\mathrm{d}_{1} \delta_{\mathrm{j}+2}^{\mathrm{n}} \text {, }
\end{aligned}
$$

Where

$$
\begin{aligned}
& \mathrm{d}_{1}=1-\frac{30 \Delta \mathrm{t}}{\mathrm{h}^{3}}-\frac{15 \Delta \mathrm{t}}{2 \mathrm{~h}} \lambda_{1}, \\
& \mathrm{~d}_{2}=26+\frac{60 \Delta \mathrm{t}}{\mathrm{h}^{3}}-\frac{150 \mathrm{a} \Delta \mathrm{t}}{2 \mathrm{~h}} \lambda_{1}, \\
& \mathrm{~d}_{3}=66, \\
& \mathrm{~d}_{4}=26-\frac{60 \Delta \mathrm{t}}{\mathrm{h}^{3}}+\frac{150 \Delta \mathrm{t}}{2 \mathrm{~h}} \lambda_{1}, \\
& \mathrm{~d}_{5}=1+\frac{30 \Delta \mathrm{t}}{\mathrm{h}^{3}}+\frac{15 \Delta \mathrm{t}}{2 \mathrm{~h}} \lambda_{1} .
\end{aligned}
$$

Substituting (13) into the difference (14), we get

$$
\zeta^{\mathrm{n}+1}\left[\begin{array}{l}
\mathrm{A}[2 \cos 2 \varphi+52 \cos \varphi+66]+ \\
\left.\sin 2 \phi\left(\begin{array}{l}
-2 \mathrm{~A}\left(\frac{30 \mathrm{a} \Delta \mathrm{t}}{\mathrm{h}^{3}}+\frac{15 \mathrm{a} \Delta \mathrm{t}}{\mathrm{h}} \lambda_{1}\right) \\
2 \mathrm{~B}\left(\frac{5 \mathrm{~b} \Delta \mathrm{t}}{\mathrm{h}} \lambda_{2}\right)
\end{array}\right)+\right] \\
\left.+\sin \phi\left(\begin{array}{l}
2 \mathrm{~A}\left(\frac{60 \mathrm{a} \Delta \mathrm{t}}{\mathrm{h}^{3}}-\frac{15 \mathrm{a} \Delta \mathrm{t}}{\mathrm{h}} \lambda_{1}\right) \\
2 \mathrm{~B}\left(\frac{150 \mathrm{~b} \Delta \mathrm{t}}{\mathrm{h}} \lambda_{2}\right)
\end{array}\right)+\right]
\end{array}\right]=
$$

Where 


$$
\left.\zeta^{\mathrm{n}}\left[\begin{array}{l}
\mathrm{A}[2 \cos 2 \varphi+52 \cos \varphi+66]+ \\
\mathrm{i}\left[\begin{array}{l}
2 \mathrm{~A} 2 \phi\left(\frac{30 \mathrm{a} \Delta \mathrm{t}}{\mathrm{h}^{3}}+\frac{15 \mathrm{a} \Delta \mathrm{t}}{\mathrm{h}} \lambda_{1}\right) \\
2 \mathrm{~B}\left(\frac{5 \mathrm{~b} \Delta \mathrm{t}}{\mathrm{h}} \lambda_{2}\right)
\end{array}\right) \\
+\sin \phi\left(\begin{array}{l}
-2 \mathrm{~A}\left(\frac{60 \mathrm{a} \Delta \mathrm{t}}{\mathrm{h}^{3}}-\frac{15 \mathrm{a} \Delta \mathrm{t}}{\mathrm{h}} \lambda_{1}\right) \\
2 \mathrm{~B}\left(\frac{150 \mathrm{~b} \Delta \mathrm{t}}{\mathrm{h}} \lambda_{2}\right)
\end{array}\right)-
\end{array}\right]\right),
$$

We get

$\mathrm{g}=\frac{\mathrm{X}+\mathrm{i} Y}{\mathrm{X}-\mathrm{i} Y}$

Where

$$
X=A[2 \cos 2 \varphi+52 \cos \varphi+66]
$$

And

$$
\mathrm{Y}=\left[\begin{array}{l}
\sin 2 \phi\left(2 \mathrm{~A}\left(\frac{30 \mathrm{a} \Delta \mathrm{t}}{\mathrm{h}^{3}}+\frac{15 \mathrm{a} \Delta \mathrm{t}}{\mathrm{h}} \lambda_{1}\right)-2 \mathrm{~B}\left(\frac{5 \mathrm{~b} \Delta \mathrm{t}}{\mathrm{h}} \lambda_{2}\right)\right) \\
+\sin \phi\left(-2 \mathrm{~A}\left(\frac{60 \mathrm{a} \Delta \mathrm{t}}{\mathrm{h}^{3}}-\frac{15 \mathrm{a} \Delta \mathrm{t}}{\mathrm{h}} \lambda_{1}\right)-2 \mathrm{~B}\left(\frac{150 \mathrm{~b} \Delta \mathrm{t}}{\mathrm{h}} \lambda_{2}\right)\right)
\end{array}\right]
$$

Similar substituting (13) into the difference (15), we get

$$
\begin{gathered}
\left.\zeta^{\mathrm{n}+1}\left[\begin{array}{c}
{[2 \cos 2 \varphi+52 \cos \varphi+66]+} \\
2 \mathrm{i}\left[\sin 2 \phi\left(\left(\frac{30 \Delta \mathrm{t}}{\mathrm{h}^{3}}+\frac{15 \Delta \mathrm{t}}{2 \mathrm{~h}} \lambda_{1}\right)\right)\right. \\
-\sin \phi\left(\left(\frac{60 \Delta \mathrm{t}}{\mathrm{h}^{3}}-\frac{15 \Delta \mathrm{t}}{2 \mathrm{~h}} \lambda_{1}\right)\right)
\end{array}\right)\right] \\
\left.\zeta^{\mathrm{n}}\left[\begin{array}{l}
\mathrm{A}[2 \cos 2 \varphi+52 \cos \varphi+66]+ \\
-2 \mathrm{i}\left[\sin 2 \phi\left(\left(\frac{30 \Delta \mathrm{t}}{\mathrm{h}^{3}}+\frac{15 \Delta \mathrm{t}}{2 \mathrm{~h}} \lambda_{1}\right)\right)\right. \\
-\sin \phi\left(\left(\frac{60 \Delta \mathrm{t}}{\mathrm{h}^{3}}-\frac{15 \Delta \mathrm{t}}{2 \mathrm{~h}} \lambda_{1}\right)\right)
\end{array}\right)\right],
\end{gathered}
$$

We get

$$
g=\frac{X-i Y}{X+i Y}
$$

Where

$$
X=[2 \cos 2 \varphi+52 \cos \varphi+66]
$$

And

$$
\mathrm{Y}=2\left[\begin{array}{l}
\sin 2 \phi\left(\left(\frac{30 \Delta \mathrm{t}}{\mathrm{h}^{3}}+\frac{15 \Delta \mathrm{t}}{2 \mathrm{~h}} \lambda_{1}\right)\right) \\
-\sin \phi\left(\left(\frac{60 \Delta \mathrm{t}}{\mathrm{h}^{3}}-\frac{15 \Delta \mathrm{t}}{2 \mathrm{~h}} \lambda_{1}\right)\right)
\end{array}\right]
$$

From (16) and (17) we get $|\mathrm{g}| \leq 1$, hence the schemes are unconditionally stable. It means that there is no restriction on the grid size, i.e. on $\mathrm{h}$ and $\Delta \mathrm{t}$, but we should choose them in such a way that the accuracy of the scheme is not degraded.

\section{Numerical Tests and Results of CKdV equation}

In this section, we present some numerical examples to test validity of our scheme for solving CKdV equation.

The norms $\mathrm{L}_{2}$-norm and $\mathrm{L}_{\infty}$-norm are used to compare the numerical solution with the analytical solution [16].

$$
\begin{aligned}
& \mathrm{L}_{2}=\left\|\mathrm{u}^{\mathrm{E}}-\mathrm{u}^{\mathrm{N}}\right\|=\sqrt{\mathrm{h} \sum_{i=0}^{N}\left(\mathrm{u}_{j}^{E}-u_{j}^{N}\right)^{2}}, \\
& \mathrm{~L}_{\infty}=\max _{j}\left|\mathrm{u}_{j}^{\mathrm{E}}-\mathrm{u}_{j}^{N}\right|, j=0,1, \cdots, N .
\end{aligned}
$$

Where $\mathrm{u}^{\mathrm{E}}$ is the exact solution $\mathrm{u}$ and $\mathrm{u}^{\mathrm{N}}$ is the approximation solution $\mathrm{U}_{\mathrm{N}}$.

And the quantities $I_{1}, I_{2}$ and $I_{3}$ are shown to measure conservation for the schemes.

$$
\begin{aligned}
& I_{1}=\int_{-\infty}^{\infty} u(x, t) d x \cong h \sum_{j=0}^{N}(U)_{j}^{n}, \\
& I_{2}=\int_{-\infty}^{\infty}\left(\begin{array}{l}
u(x, t)^{2}+ \\
\frac{2}{3} b v(x, t)^{2}
\end{array}\right) d x \cong h \sum_{j=0}^{N}\left(\begin{array}{l}
U^{2}+ \\
\frac{2}{3} b V^{2}
\end{array}\right)_{j}^{n}, \\
& \mathrm{I}_{3}=\int_{-\infty}^{\infty}\left[\begin{array}{c}
(1+\mathrm{a})\left(\begin{array}{l}
\mathrm{u}(\mathrm{x}, \mathrm{t})^{3}- \\
\frac{1}{2} \mathrm{u}_{\mathrm{x}}(\mathrm{x}, \mathrm{t})^{2}
\end{array}\right)+ \\
\mathrm{b}\left(\begin{array}{l}
\mathrm{u}(\mathrm{x}, \mathrm{t}) \mathrm{v}(\mathrm{x}, \mathrm{t})^{2}- \\
\mathrm{v}_{\mathrm{X}}(\mathrm{x}, \mathrm{t})^{2}
\end{array}\right)
\end{array}\right] \mathrm{dx \cong} \text {. } \\
& \mathrm{h} \underset{\mathrm{j}=0}{\mathrm{~N}}\left[\begin{array}{l}
(1+\mathrm{a})\left(\begin{array}{l}
\left(\mathrm{U}^{3}\right)_{\mathrm{j}}^{\mathrm{n}}- \\
\frac{1}{2}\left(\mathrm{U}_{\mathrm{x}}^{2}\right)_{\mathrm{j}}^{\mathrm{n}}
\end{array}\right)+ \\
\mathrm{b}\left(\begin{array}{l}
\left(\mathrm{UV}^{2}\right)_{\mathrm{j}}^{\mathrm{n}}- \\
\left(\mathrm{v}_{\mathrm{x}}^{2}\right)_{j}^{\mathrm{n}}
\end{array}\right],
\end{array}\right]
\end{aligned}
$$

Now we can studying our scheme from these problems.

\subsection{Single soliton}

Consider the CKdV equation (1) and (2) with the following initial and boundary conditions:

$\mathrm{u}(\mathrm{x}, 0)=\mathrm{v}(\mathrm{x}, 0)=0, \quad \mathrm{a} \leq \mathrm{x} \leq \mathrm{b}$.

And

$$
\begin{array}{ll}
\mathrm{u}(\mathrm{a}, \mathrm{t})=0, & \mathrm{u}(\mathrm{b}, \mathrm{t})=0, \\
\mathrm{v}(\mathrm{a}, \mathrm{t})=0, & \mathrm{v}(\mathrm{b}, \mathrm{t})=0, \\
\mathrm{u}_{\mathrm{X}}(\mathrm{a}, \mathrm{t})=0, & \mathrm{u}_{\mathrm{X}}(\mathrm{b}, \mathrm{t})=0, \\
\mathrm{v}_{\mathrm{X}}(\mathrm{a}, \mathrm{t})=0, & \mathrm{v}_{\mathrm{X}}(\mathrm{b}, \mathrm{t})=0, \quad 0 \leq \mathrm{t} \leq \mathrm{T} .
\end{array}
$$

The exact solution is 
$u(x, t)=2 \lambda^{2} \operatorname{sech}^{2}(\xi)$,

$\mathrm{v}(\mathrm{x}, \mathrm{t})=\frac{1}{2 \sqrt{\omega}} \operatorname{sech}(\xi)$,

$\xi=\lambda\left(x-\lambda^{2} t\right)+\frac{1}{2 \log (\omega)}$,

$\omega=\frac{-b}{8(4 a+1) \lambda^{4}}, 0 \leq t \leq T, \quad a \leq x \leq b$.

Now we can take three cases with different values of the parameters $a, b$.

Case one: we can take $\mathrm{a}=0.5, \mathrm{~b}=-3, \lambda=0.5, \mathrm{~h}=0.1, \mathrm{k}=0.01$ and $-25 \leq \mathrm{x} \leq 25$

Case two: we can take $\mathrm{a}=-0.5, \mathrm{~b}=3, \lambda=0.5, \mathrm{~h}=0.1, \mathrm{k}=0.01$ and $-25 \leq \mathrm{x} \leq 25$

Case three: we can take $\mathrm{a}=-0.125, \mathrm{~b}=-3, \lambda=0.5, \mathrm{~h}=0.1, \mathrm{k}=0.01$ and $-25 \leq \mathrm{x} \leq 25$.

Then we can studying case by case.

Case one:

Now, for comparison, we consider a test problem where, $\mathrm{a}=0.5, \mathrm{~b}=-3, \lambda=0.5, \quad \mathrm{~h}=0.1, \mathrm{k}=0.01$ and $-25 \leq \mathrm{x} \leq 25$. The simulations are done up to $t=5$. The invariant $I_{1}, I_{2}$ and $I_{3}$ approach to zero. Errors, also, at time 5 are satisfactorily small $\mathrm{L}_{2}$-error $=$ $1.88599 \times 10^{-6}$ and $\mathrm{L}_{\infty}$-error $=1.05770 \times 10^{-6}$ for approximation solution of $\mathrm{u}(\mathrm{x}, \mathrm{t})$ and $\mathrm{L}_{2}$-error $=2.95667 \times 10^{-5}$ and $\mathrm{L}_{\infty}$-error $=$ $8.57852 \times 10^{-5}$ for approximation solution of $\mathrm{v}(\mathrm{x}, \mathrm{t})$. Our results are recorded in Table 2 and Table 3 . The motion of solitary wave using our scheme is plotted at times $\mathrm{t}=0,5,10$ in Fig.1. These results illustrate that the scheme has a highest accuracy.

Table 2: Single Soliton (Conserved Quantities) for $t=5.0$, $\mathrm{a}=0.5, \mathrm{~b}=-3, \lambda=0.5, \mathrm{~h}=0.1, \mathrm{k}=0.01$ and $-25 \leq \mathrm{x} \leq 25$.

\begin{tabular}{llll}
\hline $\mathrm{T}$ & $\mathrm{I}_{1}$ & $\mathrm{I}_{2}$ & $\mathrm{I}_{3}$ \\
\hline 0.0 & 2.00000 & -0.33333 & -0.10000 \\
1.0 & 2.00000 & -0.33333 & -0.10000 \\
2.0 & 2.00000 & -0.33333 & -0.10000 \\
3.0 & 2.00000 & -0.33333 & -0.10000 \\
4.0 & 2.00000 & -0.33333 & -0.10000 \\
5.0 & 2.00000 & -0.33333 & -0.10000 \\
\hline
\end{tabular}

Table 3: $\mathrm{L}_{2}$ - Norm and $\mathrm{L}_{\infty}$ - Norm for $\mathrm{t}=5.0$, $\mathrm{a}=0.5, \mathrm{~b}=-3, \lambda=0.5, \mathrm{~h}=0.1, \mathrm{k}=0.01$ and $-25 \leq \mathrm{x} \leq 25$.

\begin{tabular}{clccc}
\hline $\mathrm{T}$ & $\mathrm{u}(\mathrm{x}, \mathrm{t})$ & & $\mathrm{v}(\mathrm{x}, \mathrm{t})$ & \\
& $\mathrm{L}_{2}-$ norm & $\mathrm{L}_{\infty}-$ norm & $\mathrm{L}_{2}-$ norm & $\mathrm{L}_{\infty}-$ norm \\
\hline 0.0 & 0.00000000 & 0.00000000 & 0.00000000 & 0.00000000 \\
1.0 & $6.06802 \mathrm{E}-7$ & $3.97188 \mathrm{E}-7$ & $4.43377 \mathrm{E}-6$ & $3.02357 \mathrm{E}-6$ \\
2.0 & $8.99862 \mathrm{E}-7$ & $5.47651 \mathrm{E}-7$ & $6.08938 \mathrm{E}-6$ & $3.83209 \mathrm{E}-6$ \\
3.0 & $1.18161 \mathrm{E}-6$ & $6.89839 \mathrm{E}-7$ & $7.21810 \mathrm{E}-6$ & $4.73962 \mathrm{E}-6$ \\
4.0 & $1.28135 \mathrm{E}-6$ & $6.98286 \mathrm{E}-6$ & $1.06873 \mathrm{E}-5$ & $2.21990 \mathrm{E}-6$ \\
5.0 & $1.88599 \mathrm{E}-6$ & $1.05770 \mathrm{E}-6$ & $2.95667 \mathrm{E}-5$ & $8.57852 \mathrm{E}-5$ \\
\hline
\end{tabular}

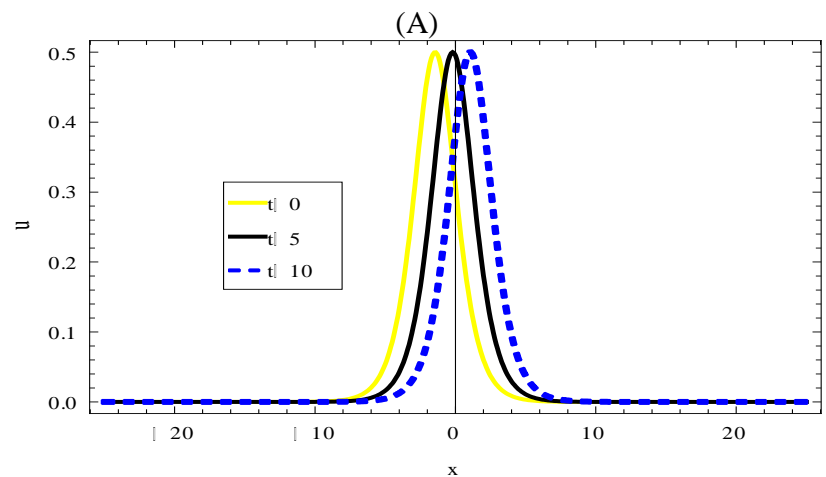

(B)

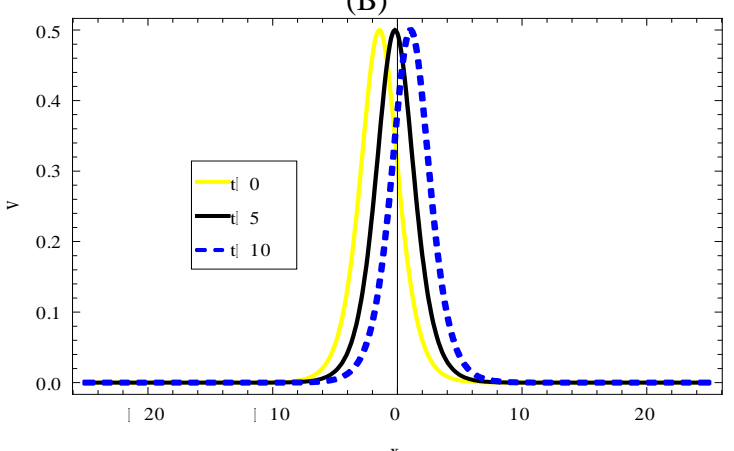

Fig. 1: Single Solitary Wave with $\mathrm{a}=0.5, \mathrm{~b}=-3, \lambda=0.5, \mathrm{~h}=0.1, \mathrm{k}=0.01 \quad$ and $\quad-25 \leq \mathrm{x} \leq 25 . \quad \mathrm{t}=0,5,10$ Respectively.

Case two:

Now, for comparison, we consider a test problem where, $\mathrm{a}=-0.5, \mathrm{~b}=3, \lambda=0.5, \mathrm{~h}=0.1, \mathrm{k}=0.01$ and $-25 \leq \mathrm{x} \leq 25$. The simulations are done up to $t=2$. The invariant $I_{1}, I_{2}$ and $I_{3}$ approach to zero. Errors, also, at time 5 are satisfactorily small $\mathrm{L}_{2}$-error = $7.00355 \times 10^{-7}$ and $\mathrm{L}_{\infty}$-error $=4.09784 \times 10^{-7}$ for approximation solution of $\mathrm{u}(\mathrm{x}, \mathrm{t})$ and $\mathrm{L}_{2}$-error $=1.77647 \times 10^{-6}$ and $\mathrm{L}_{\infty}$-error $=$ $1.30660 \times 10^{-6}$ for approximation solution of $\mathrm{v}(\mathrm{x}, \mathrm{t})$. Our results are recorded in Table 4 and Table 5 . The motion of solitary wave using our scheme is plotted at times $t=0,5,10$ in Fig.2. These results illustrate that the scheme has a highest accuracy.

Table 4: Single Soliton (Conserved Quantities) for $\mathrm{t}=5.0$, $\mathrm{a}=-0.5, \mathrm{~b}=3, \lambda=0.5, \mathrm{~h}=0.1, \mathrm{k}=0.01$ and $-25 \leq \mathrm{x} \leq 25$.

\begin{tabular}{llll}
\hline $\mathrm{T}$ & $\mathrm{I}_{1}$ & $\mathrm{I}_{2}$ & $\mathrm{I}_{3}$ \\
\hline 0.0 & 2.00000 & 0.10000 & 0.30000 \\
1.0 & 2.00000 & 0.10000 & 0.30000 \\
2.0 & 2.00000 & 0.10000 & 0.30000 \\
3.0 & 2.00000 & 0.10000 & 0.30000 \\
4.0 & 2.00000 & 0.10000 & 0.30000 \\
5.0 & 2.00000 & 0.10000 & 0.30000 \\
\hline
\end{tabular}

Table 5: $\mathrm{L}_{2}$ - Norm and $\mathrm{L}_{\infty}-$ Norm for $\mathrm{t}=2.0$, $\mathrm{a}=-0.5, \mathrm{~b}=3, \lambda=0.5, \mathrm{~h}=0.1, \mathrm{k}=0.01$ and $-25 \leq \mathrm{x} \leq 25$.

\begin{tabular}{clccc}
$\mathrm{T}$ & $\mathrm{u}(\mathrm{x}, \mathrm{t})$ & & $\mathrm{v}(\mathrm{x}, \mathrm{t})$ & \\
& $\mathrm{L}_{2}-$ norm & $\mathrm{L}_{\infty}-$ norm & $\mathrm{L}_{2}-$ norm & $\mathrm{L}_{\infty}-$ norm \\
\hline 0.0 & 0.00000000 & 0.00000000 & 0.00000000 & 0.00000000 \\
0.5 & $4.15923 \mathrm{E}-7$ & $2.96761 \mathrm{E}-7$ & $9.64209 \mathrm{E}-7$ & $7.91019 \mathrm{E}-7$ \\
1.0 & $5.59712 \mathrm{E}-7$ & $3.42588 \mathrm{E}-7$ & $1.34329 \mathrm{E}-6$ & $1.03821 \mathrm{E}-6$ \\
1.5 & $6.41255 \mathrm{E}-7$ & $3.75058 \mathrm{E}-7$ & $1.57957 \mathrm{E}-6$ & $1.10122 \mathrm{E}-6$ \\
2.0 & $7.00355 \mathrm{E}-7$ & $4.09784 \mathrm{E}-7$ & $1.77647 \mathrm{E}-6$ & $1.30660 \mathrm{E}-6$ \\
\hline
\end{tabular}

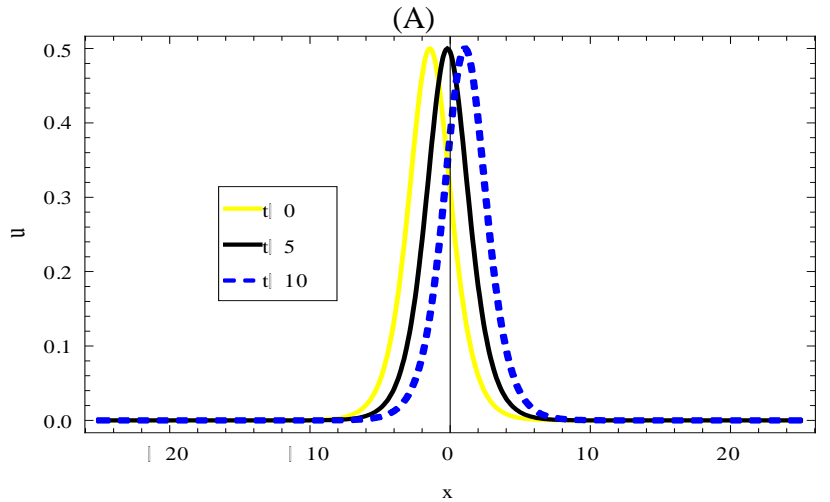


(B)

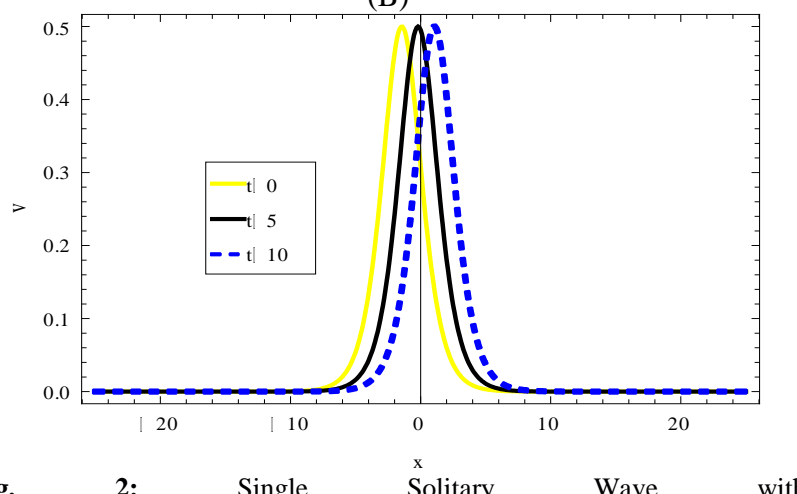

$\mathrm{a}=-0.5, \mathrm{~b}=3, \lambda=0.5, \mathrm{~h}=0.1, \mathrm{k}=0.01$ and $-25 \leq \mathrm{x} \leq 25 . \quad \mathrm{t}=0,5,10$ Respectively.

Case three:

Now, for comparison, we consider a test problem where, $\mathrm{a}=-0.125, \mathrm{~b}=-3, \lambda=0.5, \mathrm{~h}=0.1, \mathrm{k}=0.01$ and $-25 \leq \mathrm{x} \leq 25$. The simulations are done up to $t=1.5$. The invariant $\mathrm{I}_{1}, \mathrm{I}_{2}$ and $\mathrm{I}_{3}$ approach to zero. Errors, also, at time 5 are satisfactorily small $\mathrm{L}_{2}$ -error $=2.97019 \times 10^{-6}$ and $\mathrm{L}_{\infty}$-error $=8.94033 \times 10^{-6}$ for approximation solution of $\mathrm{u}(\mathrm{x}, \mathrm{t})$ and $\mathrm{L}_{2}$-error $=9.91664 \times 10^{-7}$ and $\mathrm{L}_{\infty}$ error $=7.21469 \times 10^{-7}$ for approximation solution of $\mathrm{v}(\mathrm{x}, \mathrm{t})$. Our results are recorded in Table 6 and Table 7. The motion of solitary wave using our scheme is plotted at times $t=0,5,10$ in Fig.3. These results illustrate that the scheme has a highest accuracy.

Table 6: Single Soliton (Conserved Quantities) for $\mathrm{t}=5.0$,

\begin{tabular}{llcc}
\multicolumn{3}{c}{$\mathrm{a}=-0.125, \mathrm{~b}=-3, \lambda=0.5, \mathrm{~h}=0.1, \mathrm{k}=0.01$ and $-25 \leq \mathrm{x} \leq 25}$. \\
\hline $\mathrm{T}$ & $\mathrm{I}_{1}$ & $\mathrm{I}_{2}$ & $\mathrm{I}_{3}$ \\
\hline 0.0 & 2.00000 & 0.50000 & 0.15000 \\
1.0 & 2.00000 & 0.50000 & 0.15000 \\
2.0 & 2.00000 & 0.50000 & 0.15000 \\
3.0 & 2.00000 & 0.50000 & 0.15000 \\
4.0 & 2.00000 & 0.50000 & 0.15000 \\
5.0 & 2.00000 & 0.50000 & 0.15000 \\
\hline
\end{tabular}

Table 7: $\mathrm{L}_{2}$ - Norm and $\mathrm{L}_{\infty}$ - Norm for $\mathrm{t}=1.5$, $\mathrm{a}=-0.125, \mathrm{~b}=-3, \lambda=0.5, \mathrm{~h}=0.1, \mathrm{k}=0.01$ and $-25 \leq \mathrm{x} \leq 25$.

\begin{tabular}{ccccc}
\hline $\mathrm{T}$ & $\begin{array}{l}\mathrm{u}(\mathrm{x}, \mathrm{t}) \\
\mathrm{L}_{2}-\text { norm }\end{array}$ & $\mathrm{L}_{\infty}-$ norm & $\mathrm{L}_{2}-$ norm & $\mathrm{L}_{\infty}-$ norm \\
\hline 0.0 & 0.00000000 & 0.00000000 & 0.00000000 & 0.00000000 \\
0.5 & $1.65004 \mathrm{E}-7$ & $1.42865 \mathrm{E}-7$ & $6.06562 \mathrm{E}-7$ & $5.17435 \mathrm{E}-7$ \\
1.0 & $2.92610 \mathrm{E}-7$ & $1.88360 \mathrm{E}-7$ & $8.43919 \mathrm{E}-7$ & $6.79108 \mathrm{E}-7$ \\
1.5 & $2.97019 \mathrm{E}-6$ & $8.94033 \mathrm{E}-6$ & $9.91664 \mathrm{E}-7$ & $7.21469 \mathrm{E}-7$ \\
\hline
\end{tabular}

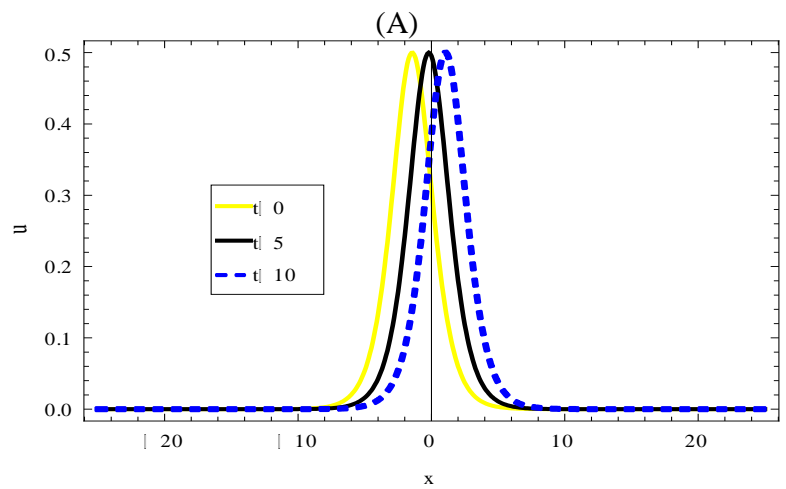

(B)

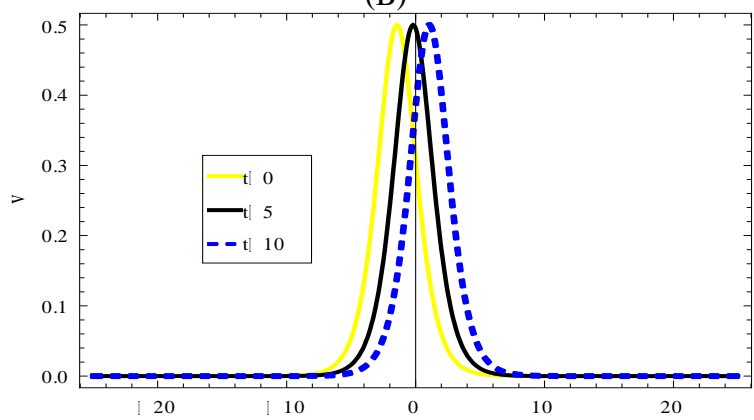

Fig. 3: Single $\quad \stackrel{x}{\text { Solitary }}$ Wave with $\mathrm{a}=-0.125, \mathrm{~b}=-3, \lambda=0.5, \mathrm{~h}=0.1, \mathrm{k}=0.01$ and $-25 \leq \mathrm{x} \leq 25 . \mathrm{t}=0,5,10$ Respectively.

Table 8: Comparison of Numerical Results of the Problem (1) with the Results Obtained from [16] and [6] for the Variable $\mathrm{u}$ and $\mathrm{v}$ with, $\mathrm{a}=0.5, \mathrm{~b}=-3, \lambda=0.5,-25 \leq \mathrm{x} \leq 25$ at $\mathrm{t}=1$.

\begin{tabular}{lllll}
\hline & $\mathrm{u}(\mathrm{x}, \mathrm{t})$ & & $\mathrm{v}(\mathrm{x}, \mathrm{t})$ & \\
Schemes at $\mathrm{t}=1$ & $\mathrm{~L}_{2}{ }^{-}$ & $\mathrm{L}_{\infty}{ }^{-}$ & $\mathrm{L}_{2}-$ & $\mathrm{L}_{\infty}{ }^{-}$ \\
& norm & norm & norm & norm \\
\hline our scheme & & & & \\
Petrov-Galerkin [6] & 0.000000 & 0.000000 & 0.000004 & 0.000003 \\
Product approxima- & - & 0.000051 & - & 0.000027 \\
tion [6] & - & 0.000014 & - & 0.000019 \\
$\begin{array}{l}\text { Collocation (Ismail } \\
\text { [4]) }\end{array}$ & - & 0.000000 & - & 0.000003 \\
\hline
\end{tabular}

In table 8 we show that our results are related with the results in [4] and better than the results in [6].

\subsection{Interaction of two solitary waves}

The interaction of two solitary waves having different amplitudes and traveling in the same direction is illustrated. We consider $\mathrm{CKdV}$ equation with initial conditions given by the linear sum of two well separated solitary waves of various amplitudes

$$
\begin{aligned}
& \mathrm{u}_{\mathrm{j}}(\mathrm{x}, \mathrm{t})=2 \lambda_{\mathrm{j}}^{2} \operatorname{sech}^{2}\left(\xi_{\mathrm{j}}\right), \\
& \mathrm{v}_{\mathrm{j}}(\mathrm{x}, \mathrm{t})=\frac{1}{2 \sqrt{\omega_{j}}} \operatorname{sech}\left(\xi_{\mathrm{j}}\right), \\
& \xi_{\mathrm{j}}=\lambda_{\mathrm{j}}\left(\mathrm{x}-\mathrm{y}_{\mathrm{j}}\right)+\frac{1}{2 \log \left(\omega_{\mathrm{j}}\right)}, \\
& \omega_{\mathrm{j}}=\frac{-\mathrm{b}}{8(4 \mathrm{a}+1) \lambda_{\mathrm{j}}{ }^{4}}, \quad \mathrm{a} \leq \mathrm{x} \leq \mathrm{b} .
\end{aligned}
$$

Where $\mathrm{j}=1,2, \lambda_{\mathrm{j}}$ and $\mathrm{y}_{\mathrm{j}}$ are arbitrary constants. In our computational work. Now, we choose $\lambda_{1}=0.9, \lambda_{2}=0.5, y_{1}=-5, y_{2}=5$, $\mathrm{a}=0.5, \mathrm{~b}=-3, \mathrm{~h}=0.1, \mathrm{k}=0.01$ with interval $[-25,25]$. In Figs. 3 and 4 , the interactions of these solitary waves are plotted at different time levels. 


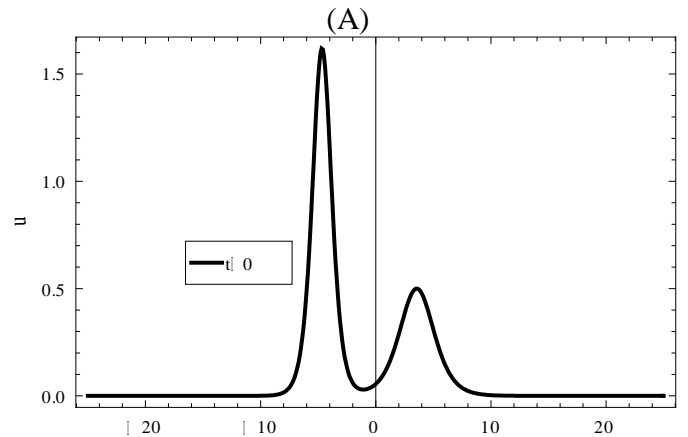

(B)

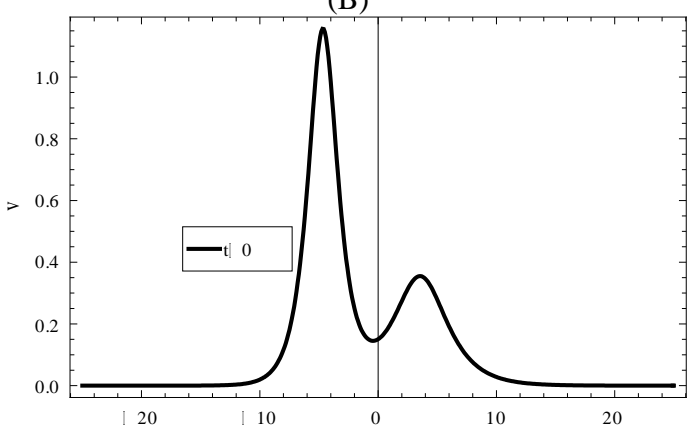

Fig. 3: Interaction Two Solitary Waves with $\lambda_{1}=0.9, \lambda_{2}=0.5, \mathrm{y}_{1}=-5, \mathrm{y}_{2}=5, \mathrm{~h}=0.1, \quad \mathrm{k}=0.01,-25 \leq \mathrm{x} \leq 25$ at Time $\mathrm{t}=0$ for Values $\mathrm{u}$ and $\mathrm{v}$ Respectively.

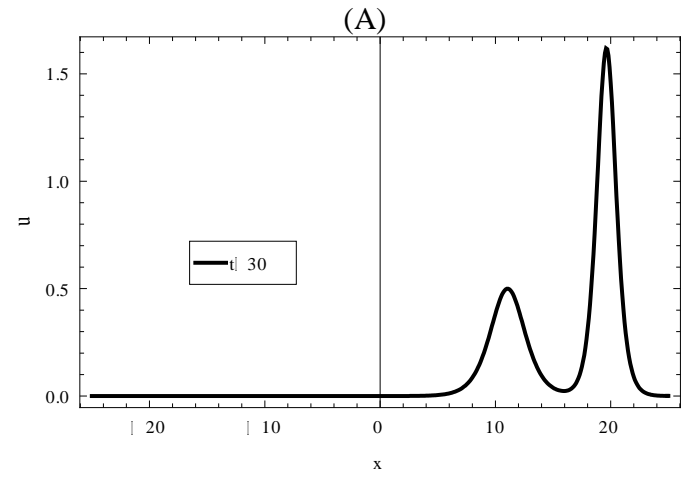

(B)

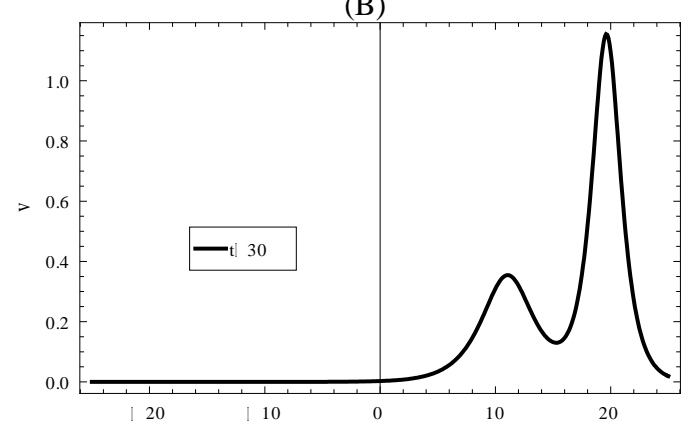

Fig. 4: Interaction Two Solitary Waves with $\lambda_{1}=0.9, \lambda_{2}=0.5, \mathrm{y}_{1}=-5, \mathrm{y}_{2}=5, \mathrm{~h}=0.1, \quad \mathrm{k}=0.01,-25 \leq \mathrm{x} \leq 25$ at Time $\mathrm{t}=30$ for Values $\mathrm{u}$ and $\mathrm{v}$ Respectively.

\subsection{Interaction of three solitary waves}

The interaction of two solitary waves having different amplitudes and traveling in the same direction is illustrated. We consider $\mathrm{CKdV}$ equation with initial conditions given by the linear sum of three well separated solitary waves of various amplitudes

$$
\begin{aligned}
& u_{j}(x, t)=2 \lambda_{j}^{2} \operatorname{sech}^{2}\left(\xi_{j}\right), \\
& v_{j}(x, t)=\frac{1}{2 \sqrt{\omega_{j}}} \operatorname{sech}\left(\xi_{j}\right), \\
& \xi_{j}=\lambda_{j}\left(x-y_{j}\right)+\frac{1}{2 \log \left(\omega_{j}\right)}, \\
& \omega_{j}=\frac{-b}{8(4 a+1) \lambda_{j}^{4}}, \quad a \leq x \leq b .
\end{aligned}
$$

Where $\mathrm{j}=1,2,3, \lambda_{\mathrm{j}}$ and $\mathrm{y}_{\mathrm{j}}$ are arbitrary constants. In our computational work. Now, we choose

$\lambda_{1}=1, \lambda_{2}=0.9, \lambda_{3}=0.8, y_{1}=-20, y_{2}=-16, y_{2}=-12$,

$\mathrm{a}=0.5, \mathrm{~b}=-3, \mathrm{~h}=0.1, \mathrm{k}=0.01$ With interval $[-25,25]$. In Figs. 5 and 6 , the interactions of these solitary waves are plotted at different time levels.
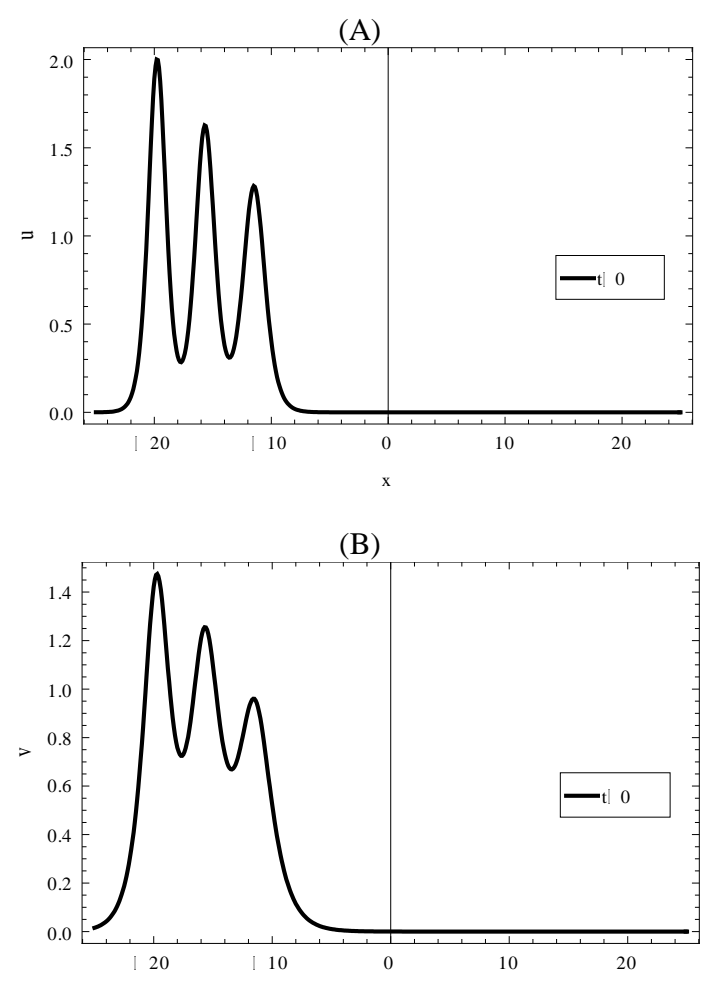

Fig. 5: Interaction Three Solitary Waves with $\lambda_{1}=1, \lambda_{2}=0.9, \lambda_{3}=0.8, \mathrm{y}_{1}=-20, \mathrm{y}_{2}=-16$,

$\mathrm{y}_{2}=-12, \mathrm{~h}=0.1, \mathrm{k}=0.01,-25 \leq \mathrm{x} \leq 25$ at Time $\mathrm{t}=0$ for Values $\mathrm{u}$ and $\mathrm{v}$ Respectively.

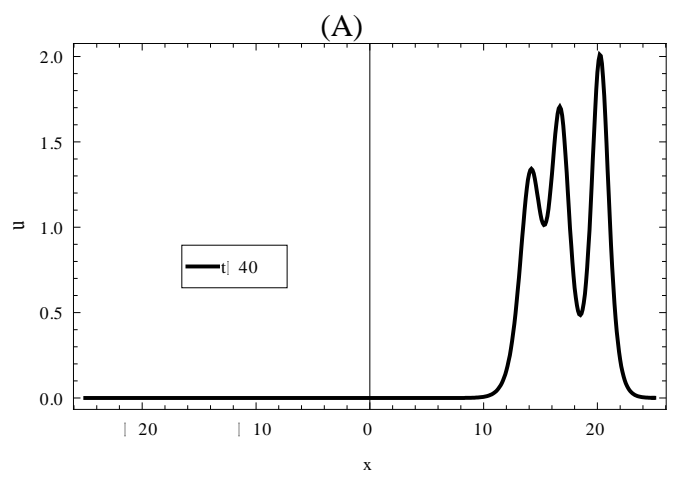


(B)

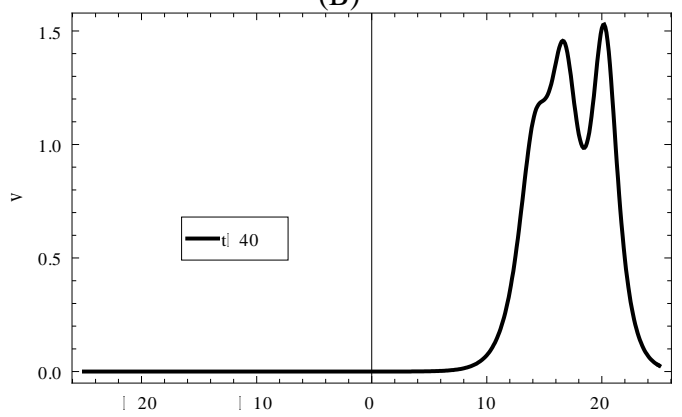

Fig. 6: Interaction Three ${ }^{\mathrm{x}}$ Solitary Waves with $\lambda_{1}=1, \lambda_{2}=0.9, \lambda_{3}=0.8, \mathrm{y}_{1}=-20, \mathrm{y}_{2}=-16$,

$\mathrm{y}_{2}=-12, \mathrm{~h}=0.1, \mathrm{k}=0.01,-25 \leq \mathrm{x} \leq 25$ at Time $\mathrm{t}=40$ for Values $\mathrm{u}$ and $\mathrm{v}$ Respectively.

\section{Conclusions}

In this paper a numerical treatment for the nonlinear CKdV equation is proposed using a collection method with the quintic Bsplines. The stability analysis of the method is shown to be unconditionally stable. We make linearization for the nonlinear term. We tested our schemes through a single solitary wave in which the analytic solution is known, then extend it to study the interaction of solitons where no analytic solution is known during the interaction. The accuracy of our scheme was shown by calculating error norms $\mathrm{L}_{2}$ and $\mathrm{L}_{\infty}$ this document can be used as a template for Microsoft Word versions 6.0 or later. Do not submit papers written with other editors than MS Word, it will not be accepted for review. Save the files to be compatible with many versions of MSWord (avoid other document extension than *.doc, *.docx or *.rtf). Do not submit papers without performing a carefully spellcheck and English language grammar check. The style from these instructions will adjust your fonts and line spacing. Please do not change the font sizes or line spacing to squeeze more text into a limited number of pages.

\section{References}

[1] R. Hirota and J. Satsuma, "Soliton solutions of a coupled Korteweg-de Vries equation," Physics Letters a, vol. 85, no. 8-9, pp. 407-408, 1981. http://dx.doi.org/10.1016/0375-9601(81)90423$\underline{0}$.

[2] A. A. Halim, S. P. Kshevetskii, and S. B. Leble, "Numerical integration of a coupled Korteweg-de Vries system," Computers \& Mathematics with Applications, vol. 45, no. 4-5, pp. 581-591, 2003. http://dx.doi.org/10.1016/S0898-1221(03)00018-X.

[3] A. A. Halim and S. B. Leble, "Analytical and numerical solution of a coupled KdV- MKdV System," Chaos, Solitons and Fractals, vol. 19, no. 1, pp. 99-108, 2004. http://dx.doi.org/10.1016/S09600779(03)00085-7.

[4] M. S. Ismail, "Numerical solution of a coupled Korteweg-de Vries equations by collocation method," Numerical Methods for Partial Differential Equations, vol. 25, no. 2, pp. 275-291, 2009. http://dx.doi.org/10.1002/num.20343.

[5] D. Kaya and I. E. Inan, "Exact and numerical traveling wave solutions for nonlinear coupled equations using symbolic computation," Applied Mathematics and Computation, vol. 151, no. 3, pp. 775787, 2004. http://dx.doi.org/10.1016/S0096-3003(03)00535-6.

[6] M. S. Ismail and H. A. Ashi, "A Numerical Solution for HirotaSatsuma Coupled KdV Equation," Abstract and Applied Analysis, Volume 2014, 9 pages, 2014.

[7] L. M. B. Assas, "Variational iteration method for solving coupledKdV equations," Chaos, Solitons and Fractals, vol. 38, no. 4, pp. 1225-1228, 2008. S. Abbasbandy, "The application of homotopy analysis method to solve a generalized http://dx.doi.org/10.1016/j.chaos.2007.02.012.

[8] Hirota-Satsuma coupled KdV equation," Physics Letters A: General, Atomic and Solid State Physics, vol. 361, no. 6, pp. 478-483, 2007.
[9] A. Wazwaz, "The KdV equation," in Handbook of Differential Equations: Evolutionary Equations. VOL. IV, Handb. Differ. Equ., pp. 485-568, Elsevier/North- Holland, Amsterdam, the Netherlands, 2008. http://dx.doi.org/10.1016/S1874-5717(08)00009-1.

[10] S. Kutluay and Y.Ucar, "A quadratic B-spline Galerkin approach for solving a coupled KdV equation," Mathematical Modelling and Analysis, vol. 18, no. 1, pp.

[11] M. S. Ismail, "Numerical solution of coupled nonlinear Schr"odinger equation by Galerkin method," Mathematics and Computers in Simulation, vol. 78, no. 4, pp. 532- 547, 2008 http://dx.doi.org/10.1016/j.matcom.2007.07.003.

[12] M. S. Ismail and T. R. Taha, "A linearly implicit conservative scheme for the coupled nonlinear Schr"odinger equation," Mathematics and Computers in Simulation, vol. 74, no. 4-5, pp. 302-311, 2007. http://dx.doi.org/10.1016/j.matcom.2006.10.020.

[13] M. S. Ismail and S. Z. Alamri, "Highly accurate finite difference method for coupled nonlinear Schr"odinger equation," International Journal of Computer Mathematics, vol. 81, no. 3, pp. 333-351, 2004. http://dx.doi.org/10.1080/00207160410001661339.

[14] T. S. EL-Danaf, K. R. Raslan and Khalid K. Ali," collocation method with cubic B- Splines for solving the GRLW equation", Int J. of Num. Meth. And Appl. Vol. 15 (1), pp. 39-59, 2016.

[15] Rubin SG, Graves RA. Cubic spline approximation for problems in fluid mechanics. Nasa TR R-436. Washington, DC; 1975.

[16] T. S. EL-Danaf, K. R. Raslan and Khalid K. Ali,"New Numerical treatment for the Generalized Regularized Long Wave Equation based on finite difference scheme", Int. J. of S. Comp. and Eng. (IJSCE)', 4, 16-24, 2014 